\title{
(T, I, F)-Neutrosophic Structures
}

\author{
Florentin Smarandache ${ }^{1, a^{*}}$ \\ ${ }^{1}$ University of New Mexico, 705 Gurley Ave., Gallup, NM 87301, USA \\ a smarand@unm.edu
}

Keywords: neutrosophy; neutrosophic structures; algebraic structures; neutrosophic bi-algebraic structures; neutrosophic $\mathrm{N}$-algebraic structures; neutrosophic groupoid; neutrosophic geometry; neutrosophic logic; neutrosophic axiom; indeterminacy.

\begin{abstract}
In this paper we introduce for the first time a new type of structures, called (T, I, F)Neutrosophic Structures, presented from a neutrosophic logic perspective, and we show particular cases of such structures in geometry and in algebra.
\end{abstract}

\section{Introduction}

In any field of knowledge, each structure is composed from two parts: a space, and a set of axioms (or laws) acting (governing) on it. If the space, or at least one of its axioms (laws), has some indeterminacy, that structure is a (T, I, F)-Neutrosophic Structure.

The (T, I, F)-Neutrosophic Structures [based on the components T=truth, I=indeterminacy, $\mathrm{F}=$ falsehood] are different from the Neutrosophic Algebraic Structures [based on neutrosophic numbers of the form $a+b I$, where $I=$ indeterminacy and $\left.I^{\wedge} n=I\right]$, that we rename as Neutrosophic IAlgebraic Structures (meaning algebraic structures based on indeterminacy "I" only). But we can combine both and obtain the (T, I, F)-Neutrosophic I-Algebraic Structures, i.e. algebraic structures based on neutrosophic numbers of the form a+bI, but also having indeterminacy related to the structure space (elements which only partially belong to the space, or elements we know nothing if they belong to the space or not) or indeterminacy related to at least one axiom (or law) acting on the structure space. Then we extend them to Refined (T, I, F)-Neutrosophic Refined I-Algebraic Structures.

\section{Neutrosophic Algebraic Structures [or Neutrosophic I-Algebraic Structures]}

A previous type of neutrosophic structures was introduced in algebra by W.B. Vasantha Kandasamy and Florentin Smarandache [1-56], since 2003, and it was called Neutrosophic Algebraic Structures. Later on, more researchers joined the neutrosophic research, such as: Mumtaz Ali, A. A. Salama, Muhammad Shabir, K. Ilanthenral, Meena Kandasamy, H. Wang, Y.-Q. Zhang, R. Sunderraman, Andrew Schumann, Salah Osman, D. Rabounski, V. Christianto, Jiang Zhengjie, Tudor Paroiu, Stefan Vladutescu, Mirela Teodorescu, Daniela Gifu, Alina Tenescu, Fu Yuhua, Francisco Gallego Lupiañez, etc.

The neutrosophic algebraic structures are algebraic structures based on sets of neutrosophic numbers of the form $N=a+b I$, where $a, b$ are real (or complex) numbers, and $a$ is called the determinate part on $N$ and $b$ is called the indeterminate part of $N$, while $I=$ indeterminacy, with $m I$ $+n I=(m+n) I, O \cdot I=0, I^{n}=I$ for integer $n \geq 1$, and $I / I=$ undefined.

When $a, b$ are real numbers, then $a+b I$ is called a neutrosophic real number. While if $a, b$ are complex numbers, then $a+b I$ is called a neutrosophic complex number.

We may say "indeterminacy" for " $I$ " from $a+b I$, and "degree of indeterminacy" for " $I$ " from $(T, I$, $F$ ) in order to distinguish them.

The neutrosophic algebraic structures studied by Vasantha-Smarandache in the period 2003-2015 are: neutrosophic groupoid, neutrosophic semigroup, neutrosophic group, neutrosophic ring, neutrosophic field, neutrosophic vector space, neutrosophic linear algebras etc., which later 
(between 2006-2011) were generalized by the same researchers to neutrosophic bi-algebraic structures, and more general to neutrosophic $N$-algebraic structures.

Afterwards, the neutrosophic structures were further extended to neutrosophic soft algebraic structures by Florentin Smarandache, Mumtaz Ali, Muhammad Shabir, and Munazza Naz in 20132014. In 2015 Smarandache refined the indeterminacy $I$ into different types of indeterminacies (depending on the problem to solve) such as $I_{1}, I_{2}, \ldots, I_{p}$ with integer $p \geq 1$, and obtained the refined neutrosophic numbers of the form $N_{p}=a+b_{1} I_{1}+b_{2} I_{2}+\ldots+b_{p} I_{p}$ where $a, b_{1}, b_{2}, \ldots, b_{p}$ are real or complex numbers, and $a$ is called the determinate part of $N_{p}$, while for each $k \epsilon\{1,2, \ldots, p\} I_{k}$ is called the $k$-th indeterminate part of $N_{p}$, and for each $k \epsilon\{1,2, \ldots, p\}$, and similarly $m I_{k}+n I_{k}=(m+$ $n) I_{k}, O \cdot I_{k}=0, I_{k}{ }^{n}=I_{k}$ for integer $n \geq 1$, and $I_{k} / I_{k}=$ undefined. The relationships and operations between $I_{j}$ and $I_{k}$, for $j \neq k$, depend on each particular problem we need to solve.

Then consequently Smarandache [2015] extended the neutrosophic algebraic structures to Refined Neutrosophic Algebraic Structures [or Refined Neutrosophic I-Algebraic Structures], which are algebraic structures based on the sets of the refined neutrosophic numbers $a+b_{1} I_{1}+b_{2} I_{2}+\ldots+b_{p} I_{p}$.

\section{(T, I, F)-Neutrosophic Structures}

We now introduce for the first time another type of neutrosophic structures. These structures, in any field of knowledge, are considered from a neutrosophic logic point of view, i.e. from the truthindeterminacy-falsehood (T, I, F) values. In neutrosophic logic every proposition has a degree of truth $(\mathrm{T})$, a degree of indeterminacy $(\mathrm{I})$, and a degree of falsehood $(\mathrm{F})$, where T, I, F are standard or non-standard subsets of the non-standard unit interval $]^{-} 0,1^{+}[$. In technical applications $\mathrm{T}$, I, and $\mathrm{F}$ are only standard subsets of the standard unit interval $[0,1]$ with: ${ }^{-} 0 \leq \sup (T)+\sup (I)+\sup (F) \leq 3^{+}$ where $\sup (Z)$ means superior of the subset $Z$.

In general, each structure is composed from: a space, endowed with a set of axioms (or laws) acting (governing) on it. If the space, or at least one of its axioms, has some indeterminacy, we consider it as a (T, I, F)-Neutrosophic Structure.

Indeterminacy with respect to the space is referred to some elements that partially belong [i.e. with a neutrosophic value (T, I. F)] to the space, or their appurtenance to the space is unknown.

An axiom (or law) which deals with indeterminacy is called neutrosophic axiom (or law).

We introduce these new structures because in the world we do not always know exactly or completely the space we work in; and because the axioms (or laws) are not always well defined on this space, or may have indeterminacies when applying them.

The neutrosophic structures are used in sensor fusions employed by robots and various technologies.

\section{Refined (T, I, F)-Neutrosophic Structures [or ( $\left.\mathbf{T}_{\mathbf{j}}, \mathbf{I}_{\mathbf{k}}, \mathbf{F}_{\mathbf{l}}\right)$-Neutrosophic Structures]}

In 2013 Smarandache [76] refined the neutrosophic components $(T, I, F)$ into $\left(T_{1}, T_{2}, \ldots, T_{m} ; I_{1}\right.$, $I_{2}, \ldots, I_{p} ; F_{1}, F_{2}, \ldots, F_{r}$ ), where $m, p, r$ are integers $\geq 1$.

Consequently, we now [2015] extend the (T, I, F)-Neutrosophic Structures to $\left(T_{1}, T_{2}, \ldots, T_{m} ; I_{1}\right.$, $I_{2}, \ldots, I_{p} ; F_{1}, F_{2}, \ldots, F_{r}$ )-Neutrosophic Structures, that we called Refined $(T, I, F)$-Neutrosophic Structures [or $\left(T_{j}, I_{k}, F_{l}\right)$-Neutrosophic Structures]. These are structures whose elements have a refined neutrosophic value of the form $\left(T_{1}, T_{2}, \ldots, T_{m} ; I_{1}, I_{2}, \ldots, I_{p} ; F_{1}, F_{2}, \ldots, F_{r}\right)$ or the space has some indeterminacy of this form.

\section{(T, I, F)-Neutrosophic I-Algebraic Structures}

The (T, I, F)-Neutrosophic Structures [based on the components T=truth, I=indeterminacy, $\mathrm{F}=$ falsehood] are different from the Neutrosophic Algebraic Structures [based on neutrosophic numbers of the form $a+b I$ ]. We may rename the last ones as Neutrosophic I-Algebraic Structures (meaning: algebraic structures based on indeterminacy "I" only). 
But we can combine both of them and obtain a (T, I, F)-Neutrosophic I-Algebraic Structures, i.e. algebraic structures based on neutrosophic numbers of the form $a+b I$, but also have indeterminacy related to the structure space (elements which only partially belong to the space, or elements we know nothing if they belong to the space or not) or indeterminacy related to at least an axiom (or law) acting on the structure space.

Even more, we can generalize them to Refined (T, I, F)-Neutrosophic Refined I-Algebraic Structures, or $\left(T_{j}, I_{k}, F_{l}\right)$-Neutrosophic $I_{s}$-Algebraic Structures.

\section{Example of Refined I-Neutrosophic Algebraic Structure}

Let the indeterminacy $I$ be split into $I_{I}=$ contradiction (i.e. truth and falsehood simultaneously), $I_{2}=$ ignorance (i.e. truth or falsehood), and $I_{3}=$ unknown, and the corresponding 3-refined neutrosophic numbers of the form $a+b_{1} I_{1}+b_{2} I_{2}+b_{3} I_{3}$.

The $(\mathrm{G}, *)$ be a groupoid. Then the 3-refined I-neutrosophic groupoid is generated by $I_{1}, I_{2}, I_{3}$ and $G$ under * and it is denoted by $N_{3}(G)=\left\{\left(G U I_{1} U I_{2} U I_{3}\right), *\right\}=\left\{a+b_{1} I_{1}+b_{2} I_{2}+b_{3} I_{3} / a, b_{1}, b_{2}, b_{3}\right.$ $\in G\}$.

\section{Example of Refined (T, I, F)-Neutrosophic Structure}

Let $(T, I, F)$ be split as $\left(T_{1}, T_{2} ; I_{1}, I_{2} ; F_{1}, F_{2}, F_{3}\right)$. Let $H=\left(\left\{h_{1}, h_{2}, h_{3}\right\}\right.$, \# ) be a groupoid, where $h_{1}, h_{2}$, and $h_{3}$ are real numbers. Since the elements $h_{1}, h_{2}, h_{3}$ only partially belong to $\mathrm{H}$ in a refined way, we define a refined $(T, I, F)$-neutrosophic groupoid \{ or refined $(2 ; 2 ; 3)$-neutrosophic groupoid, since $\mathrm{T}$ was split into 2 parts, I into 2 parts, and $\mathrm{F}$ into 3 parts $\}$ as $H=\left\{h_{1}(0.1,0.1 ; 0.3\right.$, $\left.0.0 ; 0.2,0.4,0.1), h_{2}(0.0,0.1 ; 0.2,0.1 ; 0.2,0.0,0.1), h_{3}(0.1,0.0 ; 0.3,0.2 ; 0.1,0.4,0.0)\right\}$.

\section{Examples of (T, I, F)-Neutrosophic I-Algebraic Structures}

1. Indeterminate Space (due to Unknown Element). And Neutrosophic Number included.

Let $B=\{2+5 I,-I,-4, b(0,0.9,0)\}$ a neutrosophic set, which contain two neutrosophic numbers, $2+5 \mathrm{I}$ and $-I$, and we know about the element $b$ that its appurtenance to the neutrosophic set is $90 \%$ indeterminate.

2. Indeterminate Space (due to Partially Known Element). And Neutrosophic Number included.

Let $\mathrm{C}=\{-7,0,2+\mathrm{I}(0.5,0.4,0.1), 11(0.9,0,0)\}$, which contains a neutrosophic number $2+I$, and this neutrosophic number is actually only partially in $C$; also, the element 11 is also partially in $C$.

3. Indeterminacy Axiom (Law).

Let $D=[0+0 I, 1+1 I]=\{c+d I$, where $c, d \in[0,1]\}$. One defines the binary law \# in the following way: \# : $D \times D \rightarrow D, x \# y=\left(x_{1}+x_{2} I\right) \#\left(y_{1}+y_{2} I\right)=\left[\left(x_{1}+x_{2}\right) / y_{1}\right]+y_{2} I$, but this neutrosophic law is undefined (indeterminate) when $y_{1}=0$.

4. Little Known or Completely Unknown Axiom (Law).

Let us reconsider the same neutrosophic set $D$ as above. But, about the binary neutrosophic law $\Theta$ that $D$ is endowed with, we only know that it associates the neutrosophic numbers $1+I$ and $0.2+0.3 I$ with the neutrosophic number $0.5+0.4 I$, i.e. $(1+I) \Theta(0.2+0.3 I)=0.5+0.4 I$.

There are many cases in our world when we barely know some axioms (laws).

\section{Examples of Refined (T, I, F)-Neutrosophic Refined I-Algebraic Structures}

We combine the ideas from Examples 5 and 6 and we construct the following example.

Let's consider, from Example 5, the groupoid $(\mathrm{G}, *)$, where $\mathrm{G}$ is a subset of positive real numbers, and its extension to a 3-refined I-neutrosophic groupoid, which was generated by $I_{1}, I_{2}, I_{3}$ and $G$ under the law * that was denoted by $N_{3}(G)=\left\{a+b_{1} I_{1}+b_{2} I_{2}+b_{3} I_{3} / a, b_{1}, b_{2}, b_{3} \in G\right\}$.

We then endow each element from $N_{3}(G)$ with some (2; 2; 3)-refined degrees of membership/indeterminacy/nonmembership, as in Example 6, of the form $\left(T_{1}, T_{2} ; I_{1}, I_{2} ; F_{1}, F_{2}, F_{3}\right)$, and we obtain: 


$$
N_{3}(G)_{(2 ; 2 ; 3)}=\left\{a+b_{1} I_{1}+b_{2} I_{2}+b_{3} I_{3}\left(T_{1}, T_{2} ; I_{1}, I_{2} ; F_{1}, F_{2}, F_{3}\right) / a, b_{1}, b_{2}, b_{3} \in G\right\},
$$

where

$$
\begin{aligned}
& T_{1}=\frac{a}{a+b_{1}+b_{2}+b_{3}}, T_{2}=\frac{0.5 a}{a+b_{1}+b_{2}+b_{3}} ; \\
& I_{1}=\frac{b_{1}}{a+b_{1}+b_{2}+b_{3}}, I_{2}=\frac{b_{2}}{a+b_{1}+b_{2}+b_{3}} ; \\
& F_{1}=\frac{0.1 b_{3}}{a+b_{1}+b_{2}+b_{3}}, F_{2}=\frac{0.2 b_{1}}{a+b_{1}+b_{2}+b_{3}}, F 3=\frac{b_{2}+b_{3}}{a+b_{1}+b_{2}+b_{3}} .
\end{aligned}
$$

Therefore, $N_{3}(G)_{(2 ; 2 ; 3)}$ is a refined $(2 ; 2 ; 3)$-neutrosophic groupoid and a 3-refined I-neutrosophic groupoid.

\section{Neutrosophic Geometric Examples}

\section{a. Indeterminate Space.}

We might not know if a point $P$ belongs or not to a space $S$ [we write $P(0,1,0)$, meaning that $P$ 's indeterminacy is 1 , or completely unknown, with respect to $S]$.

Or we might know that a point $Q$ only partially belongs to the space $S$ and partially does not belong to the space $S$ [for example $Q(.3,0.4,0.5)$, which means that with respect to $S, Q$ 's membership is 0.3 , $Q$ 's indeterminacy is 0.4 , and $Q$ 's non-membership is 0.5 ].

Such situations occur when the space has vague or unknown frontiers, or the space contains ambiguous (not well-defined) regions.

b. Indeterminate Axiom.

Also, an axiom $(\alpha)$ might not be well defined on the space $S$, i.e. for some elements of the space the axiom $(\alpha)$ may be valid, for other elements of the space the axiom $(\alpha)$ may be indeterminate (meaning neither valid, nor invalid), while for the remaining elements the axiom $(\alpha)$ may be invalid.

As a concrete example, let's say that the neutrosophic values of the axiom $(\alpha)$ are $(0.6,0.1,0.2)=$ (degree of validity, degree of indeterminacy, degree of invalidity).

\section{(T, I, F)-Neutrosophic Geometry as a Particular Case of (T, I, F)-Neutrosophic Structures}

As a particular case of (T, I, F)-neutrosophic structures in geometry, one considers a $(T, I, F)$ Neutrosophic Geometry as a geometry which is defined either on a space with some indeterminacy (i.e. a portion of the space is not known, or is vague, confused, unclear, imprecise), or at least one of its axioms has some indeterminacy (i.e. one does not know if the axiom is verified or not in the given space).

This is a generalization of the Smarandache Geometry (SG) [57-75], where an axiom is validated and invalidated in the same space, or only invalidated, but in multiple ways. Yet the SG has no degree of indeterminacy related to the space or related to the axiom.

A simple Example of a $S G$ is the following - that unites Euclidean, Lobachevsky-Bolyai-Gauss, and Riemannian geometries altogether, in the same space, considering the Fifth Postulate of Euclid: in one region of the SG space the postulate is validated (only one parallel trough a point to a given line), in a second region of SG the postulate is invalidated (no parallel through a point to a given line - elliptical geometry), and in a third region of SG the postulate is invalidated but in a different way (many parallels through a point to a given line - hyperbolic geometry). This simple example shows a hybrid geometry which is partially Euclidean, partially Non-Euclidean Elliptic, and partially Non-Euclidean Hyperbolic. Therefore, the fifth postulate (axiom) of Euclid is true for some regions, and false for others, but it is not indeterminate for any region (i.e. not knowing how many parallels can be drawn through a point to a given line). 
We can extend this hybrid geometry adding a new space region where one does not know if there are or there are not parallels through some given points to the given lines (i.e. the Indeterminate component) and we form a more complex (T, I, F)-Neutrosophic Geometry.

\section{Neutrosophic Algebraic Examples}

1a. Indeterminate Space (due to Unknown Element).

Let the set (space) be $\mathrm{NH}=\{4,6,7,9, a\}$, where the set $\mathrm{NH}$ has an unknown element " $a$ ", therefore the whole space has some degree of indeterminacy. Neutrosophically, we write $a(0,1,0)$, which means the element $a$ is $100 \%$ unknown.

1b. Indeterminate Space (due to Partially Known Element).

Given the set $\mathrm{M}=\{3,4,9(0.7,0.1,0.3)\}$, we have two elements 3 and 4 which surely belong to $\mathrm{M}$, and one writes them neutrosophically as $3(1,0,0)$ and $4(1,0,0)$, while the third element 9 belongs only partially $(70 \%)$ to $\mathrm{M}$, its appurtenance to $\mathrm{M}$ is indeterminate (10\%), and does not belong to $\mathrm{M}$ (in a percentage of $30 \%$ ).

Suppose $\mathrm{M}$ is endowed with a neutrosophic law* defined in the following way:

$$
x_{1}\left(t_{1}, i_{1}, f_{1}\right) * x_{2}\left(t_{2}, i_{2}, f_{2}\right)=\max \left\{x_{1}, x_{2}\right\}\left(\min \left\{t_{1}, t_{2}\right\}, \max \left\{i_{1}, i_{2}\right\}, \max \left\{f_{1}, f_{2}\right\}\right),
$$

which is a neutrosophic commutative semigroup with unit element $3(1,0,0)$.

Clearly, if $x, y \in M$, then $x^{*} y \in M$. Hence the neutrosophic law $*$ is well defined.

Since $\max$ and $\min$ operators are commutative and associative, then $*$ is also commutative and associative. If $x \in M$, then $x * x=x$.

Below, examples of applying this neutrosophic law *:

$$
\begin{aligned}
& 3 * 9(0.7,0.1,0.3)=3(1,0,0) * 9(0.7,0.1,0.3)=\max \{3,9\}(\min \{1,0.7\}, \max \{0,0.1\}, \max \{0, \\
& 0.3\})=9(0.7,0.1,0.3) . \\
& 3 * 4=3(1,0,0) * 4(1,0,0)=\max \{3,4\}(\min \{1,1\}, \max \{0,0\}, \max \{0,0\})=4(1,0,0) .
\end{aligned}
$$

\section{Indeterminate Law (Operation).}

For example, let the set (space) be $\mathrm{NG}=(\{0,1,2\}, /)$, where "/" means division.

NG is a $(T, I, F)$-neutrosophic groupoid, because the operation "/" (division) is partially defined and undefined (indeterminate). Let's see:

- $2 / 1=1$, which belongs to $\mathrm{NG}$;

- $1 / 2=0.5$, which does not belongs to NG;

- $1 / 0=$ undefined (indeterminate).

So the law defined on the set NG has the properties that:

- applying this law to some elements, the results are in NG [well defined law];

- applying this law to other elements, the results are not in NG [not well defined law];

- applying this law to again other elements, the results are undefined [indeterminate law].

We can construct many such algebraic structures where at least one axiom has such behavior (such indeterminacy in principal).

\section{Websites at UNM for Neutrosophic Algebraic Structures and respectively Neutrosophic Geometries:}

http://fs.gallup.unm.edu/neutrosophy.htm; http://fs.gallup.unm.edu/geometries.htm.

\section{Acknowledgement}

The author would like to thank Mr. Mumtaz Ali, from Quaid-i-Azam University, Islamabad, Pakistan, Mr. Said Broumi, from University of Hassan II Mohammedia, Casablanca, Morocco, and Dr. W. B. Vasantha Kandasamy from Indian Institute of Technology, Chennai, Tamil Nadu, India, for their comments on the paper. 


\section{References}

\section{Neutrosophic Algebraic Structures}

[1] A. Salama \& Florentin Smarandache, Neutrosophic Crisp Set Theory, Educational Publisher, Columbus, Ohio, USA, 163 p., 2015.

[2] Florentin Smarandache, Neutrosophic Theory and its Applications, Collected Papers, Vol. I, EuropaNova, Brussels, Belgium, 480 p., 2014.

[3] Mumtaz Ali, Florentin Smarandache, Muhammad Shabir, New Research on Neutrosophic Algebraic Structures, EuropaNova, Brussels, Belgium, 333 p., 2014.

[4] Florentin Smarandache, Law of Included Multiple-Middle \& Principle of Dynamic Neutrosophic Opposition, EuropaNova \& Educational Publisher, Brussels, Belgium - Columbus, Ohio, USA, 136 p., 2014.

[5] Florentin Smarandache, Stefan Vladutescu (coordinators), Communication Neutrosophic Routes, Educational Publisher, Columbus, Ohio, USA, 217 p., 2014.

[6] F. Smarandache, Introduction to Neutrosophic Statistics, Sitech and Education Publisher, Craiova, Romania - Educational Publisher, Columbus, Ohio, USA, 123 p., 2014.

[7] Florentin Smarandache, Introduction to Neutrosophic Measure, Neutrosophic Integral, and Neutrosophic Probability, Sitech \& Educational Publisher, Craiova, Romania - Columbus, Ohio, USA, 140 p., 2013.

[8] W. B. Vasantha Kandasamy, Florentin Smarandache, Neutrosophic Interval Bialgebraic Structures, Zip Publishing, Columbus, Ohio, USA, 195 p., 2011.

[9] W.B. Vasantha Kandasamy, F. Smarandache, K, Ilanthenral, New Classes of Neutrosophic Linear Algebras, CuArt, Slatina, Romania, 286 p., 2010.

[10] Florentin Smarandache (editor), Multispace\&Multistructure. Neutrosophic Transdisciplinarity (100 Collected Papers of Sciences), Vol. IV, North-European Scientific Publishers, Hanko, Finland, 800 p., 2010.

[11] F. Smarandache, V. Christianto, Neutrosophic Logic, Wave Mechanics, and Other Stories (Selected Works: 2005-2008), Kogaion Editions, Bucharest, Romania, 129 p., 2009.

[12] W. B. Vasantha Kandasamy, Florentin Smarandache, N-Algebraic Structures and S-NAlgebraic Structures, Hexis, Phoenix, Arizona, USA, 209 p., 2006.

[13] Florentin Smarandache, D. Rabounski, L. Borissova, Neutrosophic Methods in General Relativity, Hexis, Phoenix, Arizona, USA, 78 p., 2005. - Russian translation D. Rabounski, Нейтрософские методы в Общей Теории Относительности, Hexis, Phoenix, Arizona, USA, 105 p., 2006.

[14] Florentin Smarandache, Neutrosophy. Neutrosophic Probability, Set, and Logic, American Research Press, Rehoboth, USA, 105 p., 1998. - Republished in 2000, 2003, 2005, A Unifying Field in Logics: Neutrosophic Logic. Neutrosophy, Neutrosophic Set, Neutrosophic Probability and Statistics (second, third, and respectively fourth edition), American Research Press, USA, 156 p.; Chinese translation by F. Liu, Xiquan Chinese Branch, 121 p., 2003; Сущность нейтрософии, Russian partial translation by D. Rabounski, Hexis, Phoenix, Arizona, USA, 32 p., 2006.

\section{Neutrosophic Algebraic Structures - Edited Books}

[15] Florentin Smarandache \& Mumtaz Ali - editors, Neutrosophic Sets and Systems, book series, Vol. 1-7, Educational Publisher, Columbus, Ohio, USA, 2013-2015.

\section{Neutrosophic Geometries}

[16] S. Bhattacharya, A Model to the Smarandache Geometries, in "Journal of Recreational Mathematics", Vol. 33, No. 2, p. 66, 2004-2005; - modified version in "Octogon Mathematical Magazine", Vol. 14, No. 2, pp. 690-692, October 2006.

[17] S. Chimienti and M. Bencze, Smarandache Paradoxist Geometry, in "Bulletin of Pure and Applied Sciences", Delhi, India, Vol. 17E, No. 1, 123-1124, 1998; http://www.gallup.unm. edu/ smarandache/prd-geo1.txt. 
[18] Linfan Mao, An introduction to Smarandache geometries on maps, presented at 2005 International Conference on Graph Theory and Combinatorics, Zhejiang Normal University, Jinhua, Zhejiang, P. R. China, June 25-30, 2005.

[19] H. Iseri, Smarandache Manifolds, Am. Res. Press, 2002, http://www.gallup.unm.edu/ $\sim$ smarandache /Iseri-book1.pdf

\section{Refined Neutrosophics}

[20] Florentin Smarandache, n-Valued Refined Neutrosophic Logic and Its Applications in Physics, Progress in Physics, USA, 143-146, Vol. 4, 2013. 